\title{
Crack Growth by Surface Diffusion in Viscoelastic Media
}

\author{
R. Spatschek, ${ }^{1,2}$ E. A. Brener, ${ }^{1}$ and D. Pilipenko ${ }^{1}$ \\ ${ }^{1}$ Institut für Festkörperforschung, Forschungszentrum Jülich, D-52425 Jülich, Germany \\ ${ }^{2}$ Physics Department and Center for Interdisciplinary Research on Complex Systems, Northeastern University, \\ Boston, Massachusetts 02115, USA
}

(Received 6 March 2008; published 10 November 2008)

\begin{abstract}
We discuss steady state crack growth in the spirit of a free boundary problem. It turns out that mode I and mode III situations are very different from each other: In particular, mode III exhibits a pronounced transition towards unstable crack growth at higher driving forces, and the behavior close to the Griffith point is determined entirely through crack surface dissipation, whereas in mode I the fracture energy is renormalized due to a remaining finite viscous dissipation. Intermediate mixed-mode scenarios allow steady state crack growth with higher velocities than for pure mode I.
\end{abstract}

DOI: 10.1103/PhysRevLett.101.205501

The growth of cracks is an important subject in solid state physics and materials science, still lacking a convincing physical description in many respects. It links macroscopic material properties to microscopic effects in the tiny tip region and raises the important question which features of crack growth are generic and can be attributed to larger classes of materials. Here, the Asaro-Tiller-Grinfeld instability (ATG) $[1,2]$, which is usually understood as the morphological instability of a uniaxially stressed surface or interface, turns out to be strongly related to the problem of crack growth beyond the Griffith point: Both are based on the same counterplay between a release of elastic energy and an increase of surface or fracture energy. Nevertheless, it is known that the ATG instability leads to a breakdown of the physical description in the framework of static elasticity, as the unstable solid forms deep grooves, which, after a finite time, advance with infinitely high velocity and vanishing tip radius. The reason for this breakdown is the absence of an additional microscopic length scale for selection of a cracklike tip radius. Hence, understanding the selection of a crack tip radius in fracture has important implications also for the stability of stressed surfaces.

One of the central questions for any crack model is the role of dissipation, which is directly connected to the quest for selection mechanisms for a tip scale. The elastic loading, which is applied far away from the crack tip, is usually only partially used to create the (macroscopically visible) crack surfaces; especially for higher propagation speeds a microbranching instability can significantly increase the fracture energy [3]. Oscillatory instabilities can also increase dissipation in thin samples [4]. This already indicates that the aspect of dimensionality is important for a full understanding of crack propagation, and we therefore investigate here different modes of loading. Although we have demonstrated that even pure dynamical linear elasticity can regularize the singular crack tip [5], it is natural to assume that deviations from a pure elastic behavior can play a crucial role, which can also contribute to dissipation;
PACS numbers: 62.20.mt, 46.15. $-\mathrm{x}, 46.50 .+\mathrm{a}, 47.54 .-\mathrm{r}$

plasticity is an important example [6], but still a full description has not yet been achieved there. It is obvious that a very detailed modeling of the tip region is required to investigate these different effects, which include a selfconsistent selection of the crack shape itself.

To address these important questions, we propose a description of crack propagation in the spirit of interfacial pattern formation processes by inclusion of viscoelastic effects. This picture goes beyond the usual small scale yielding that is frequently used in the modeling of brittle fracture and includes two dissipative mechanisms: First, there is dissipation directly at the crack surface; the incoming flow of elastic energy is partially converted to surface energy in order to advance the crack, and the remaining part is converted to heat. Second, an extended zone of viscous dissipation is formed around the crack. We note that this problem is quite complicated as the shape of the crack, its velocity and the distribution between viscous and interfacial dissipation have to be determined selfconsistently.

Viscous dissipation in mode I fracture has been discussed in the literature, and although our results qualitatively agree, our model makes a further step as it introduces this effect as a way to intrinsically regularize the tipsingularity by selection of the crack tip radius. In contrast, other models assume a Barenblatt crack tip model or similar ad-hoc regularization criteria. For details see, for example, [7,8], and references therein.

For simplicity we assume that the system obeys a translational invariance in one direction; thus, it is effectively two dimensional. We assume an isotropic linear viscoelastic medium, $u_{i}$ and $\epsilon_{i k}$ are displacement and strain, respectively. The total stress, $\sigma_{i k}=\sigma_{i k}^{(\mathrm{el})}+\sigma_{i k}^{(\mathrm{vis})}$, is decomposed into the elastic stress, which is given by Hooke's law (with elastic modulus $E$, Poisson ratio $\nu$ ),

$$
\sigma_{i k}^{(\mathrm{el})}=\frac{E}{1+\nu}\left[\epsilon_{i k}+\frac{\nu}{1-2 \nu} \delta_{i k} \epsilon_{l l}\right],
$$

and the viscoelastic stress [9] 


$$
\sigma_{i k}^{(\mathrm{vis})}=2 \eta\left[\dot{\epsilon}_{i k}-\delta_{i k} \dot{\epsilon}_{l l} / 3\right]+\zeta \dot{\epsilon}_{l l} \delta_{i k},
$$

which is related to the displacement rate through the viscosities $\eta$ and $\zeta$. Since we concentrate here on slow fracture with velocities far below the Rayleigh speed, the assumption of static viscoelasticity is legitimate, thus $\partial \sigma_{i k} / \partial x_{k}=0$. On the crack contour, the total normal and shear stresses have to vanish, $\sigma_{n n}=\sigma_{n s}=0$, with the interface normal and tangential directions $n$ and $s$. The driving force for crack propagation is given by the chemical potential [10]

$$
\mu_{s}=\Omega\left(\sigma_{i j}^{(\mathrm{el})} \epsilon_{i j} / 2-\gamma \kappa\right),
$$

with $\gamma$ being the interfacial energy per unit area and $\Omega$ the atomic volume; the interface curvature $\kappa$ is positive for a convex crack shape. Surface diffusion leads to the following expression for the normal velocity at each interface point

$$
v_{n}=-\frac{D}{\gamma \Omega} \frac{\partial^{2} \mu_{s}}{\partial s^{2}},
$$

with the surface diffusion constant $D$ (dimension $\mathrm{m}^{4} / \mathrm{s}$ ). Notice that $\tau_{0}:=2 \eta(1+\nu) / E$ defines a time scale; thus, $\left(D \tau_{0}\right)^{1 / 4}$ defines a length scale parameter which ultimately leads to selection of the tip scale. In the general case, another time scale (which does not differ significantly from $\tau_{0}$ ) is set similarly by the viscous coefficient $\zeta$, and we discuss the specific case that these time scales are equal, i.e., $\zeta=2 \eta[\nu /(1-2 \nu)+1 / 3]$. Of course, this simplification is only relevant for mode I fracture, as the second scale does not appear in mode III. Altogether, the above set of equations fully defines the problem.

We note that for steady state growth with velocity $v$, the last equation can be integrated once, and we obtain

$$
v y=\frac{D}{\gamma \Omega} \frac{\partial \mu_{s}}{\partial s} .
$$

We illustrate the procedure to solve the movingboundary viscoelastic problem for mode III fracture in the steady state regime; for mode I loadings a similar approach can be used, which will be explained in detail elsewhere. The symmetric crack is located in the $x y$ plane and propagates in positive $x$ direction with velocity $v$. Then Newton's equation reads

$$
\nabla^{2}\left(u_{z}+\tau_{0} \dot{u}_{z}\right)=0
$$

from which we obtain by differentiation $\nabla^{2} \sigma_{x z}=$ $\nabla^{2} \sigma_{y z}=0$. We represent the total stress through an analytical complex potential $\Sigma$ with $\sigma_{x z}=\Im(\Sigma)$ and $\sigma_{y z}=$ $\Re(\Sigma)$. For steady state growth, $\sigma_{i z}^{(\mathrm{vis})}=-v \tau_{0} \sigma_{i z, x}^{(\mathrm{el})}$, and we therefore make a similar ansatz for the representation of the elastic fields through an analytical function $\Sigma^{\text {(el) }}$, $\sigma_{x z}^{(\mathrm{el})}=\Im\left(\Sigma^{(\mathrm{el})}\right)$ and $\sigma_{y z}^{(\mathrm{el})}=\mathfrak{R}\left(\Sigma^{(\mathrm{el})}\right)$. This also guarantees the integrability of the strain field. The force balance Eq.
(6) is then satisfied for solutions of the complex differential equation

$$
\Sigma^{(\mathrm{el})}-v \tau_{0} \frac{d}{d z} \Sigma^{(\mathrm{el})}=\Sigma,
$$

with $z=x+i y$. For the total stress we use a multipole expansion with a branch cut along the negative real axis,

$$
\Sigma=\mu \sum_{m=1}^{M} A_{m} z^{1 / 2-m}
$$

with real coefficients $A_{i}$. The main mode $m=1$ is related to the stress intensity factor, $A_{1}=K_{\mathrm{III}} / \mu(2 \pi)^{1 / 2}(\mu=$ $E / 2(1+\nu)$ is the shear modulus), which we assume to be given as for "fixed grips" conditions for crack growth in a strip. The other coefficients are adjusted such that the boundary condition on the (extended) crack shape, $\sigma_{n z}=$ 0 , is satisfied. To this end we minimize the residual stress functional $\int \sigma_{n z}^{2} d s$ (integrated along the crack contour) with respect to the expansion coefficients and solve the arising linear problem numerically for a known crack shape. We restrict the calculation to a finite number of modes $M$ in such a way that the final result does not change noticeably if the accuracy is increased. Equation (7) can now be solved for each mode, and we obtain

$$
\Sigma^{(\mathrm{el})}=\mu \sum_{m=1}^{M} A_{m} \Sigma_{m}^{(\mathrm{el})}
$$

with the recursion relation

$$
\begin{gathered}
\Sigma_{1}^{(\mathrm{el})}=\pi^{1 / 2}\left(v \tau_{0}\right)^{-1 / 2} \exp \left(\frac{z}{v \tau_{0}}\right) \operatorname{erfc} \sqrt{\frac{z}{v \tau_{0}}} \\
\Sigma_{m+1}^{(\mathrm{el})}=\frac{1}{(m-1 / 2) v \tau_{0}}\left[z^{1 / 2-m}-\Sigma_{m}^{(\mathrm{el})}\right] .
\end{gathered}
$$

The integration constant is chosen such that far away from the crack tip the purely elastic behavior is retained. These expressions can then be used to obtain the strain from Hooke's law, and-after integration-the displacement field $u_{z}$.

The strategy of solution is therefore as follows: First, for known crack shape, the total stress problem is solved in the spirit of Eq. (8), delivering the coefficients of expansion $A_{i}$. They, together with a given crack speed are used to determine the elastic stress field according to Eqs. (9)-(11). Then, in the next step, the chemical potential (3) can be computed using Hooke's law. Finally, the steady state Eq. (5) is a nonlocal and nonlinear relation which is used to determine a new guess for the crack shape and velocity. With them, the whole procedure is iterated until a selfconsistent solution is found.

We define a dimensionless driving force

$$
\Delta=\Delta_{\mathrm{I}}+\Delta_{\mathrm{III}}=\frac{1-\nu^{2}}{2 E \gamma} K_{\mathrm{I}}^{2}+\frac{1}{4 \mu \gamma} K_{\mathrm{III}}^{2},
$$




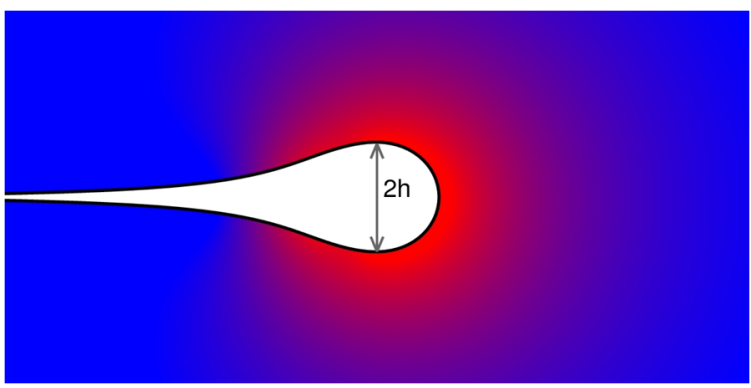

FIG. 1 (color online). Shape of a mode III crack for $\Delta=2.5$ in the steady state regime. The total incoming elastic energy flux is converted into surface energy, surface dissipation and viscous bulk dissipation, which is localized to the scale $v \tau_{0}$ around the crack tip (visualized by the color coding).

where we already included the possibility of mixed-mode loading, and $\Delta=1$ is the Griffith point. From now on, we set $\nu=1 / 3$. Figure 1 shows a typical steady state crack shape for mode III loading in the reference frame (Lagrangian coordinates); i.e., the elastic displacement is not included. First, we clearly see that the crack tip scale is selected self-consistently, and the finite time cusp singularity of the ATG instability does not occur. Therefore, the presence of viscous bulk dissipation is a way to cure this well-known problem. Second, it is important that far behind the crack tip the opening decays to zero, which is a consequence of mass conservation, as expressed by the equation of motion for surface diffusion (4). Diffusive transport is therefore restricted to the tip region, and no long-range transport is required. Qualitatively, the crack shapes for mode I look very similar.

Interestingly, the propagation velocity differs quite significantly for mode I and mode III fracture, as shown in Fig. 2: For mode III, the crack speed increases with the driving force, until it reaches a maximum at $\Delta \approx 3.5$, then it decreases, and obviously steady state solutions do not

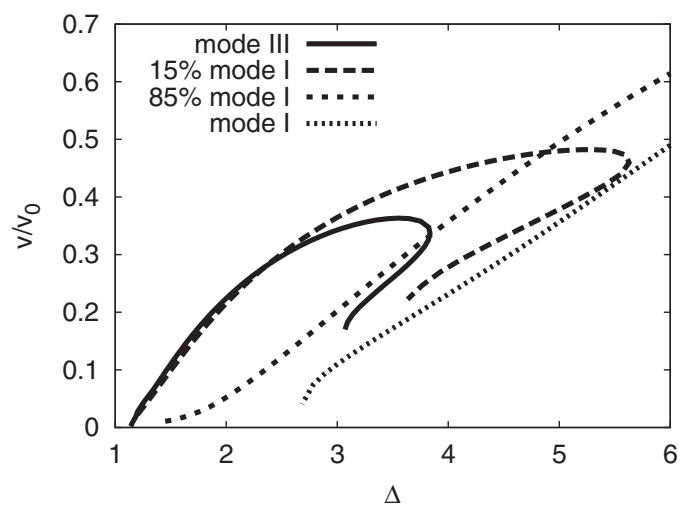

FIG. 2. Steady state propagation velocity as a function of the driving force for pure mode III and mode I fracture. Additionally, mixed-mode situations with $\Delta_{I} / \Delta=0.15$ and $\Delta_{I} / \Delta=0.85$ are displayed. The velocity scale is $v_{0}=$ $\left(D \tau_{0}^{-3}\right)^{1 / 4}$. exist beyond the point $\Delta \approx 3.8$. There, the stable branch merges with another (unstable) solution on which the tip curvature becomes negative. Beyond the bifurcation point we expect crack branching, in analogy to our findings for fast brittle fracture [5]. We note, however, that the scale of the critical crack speed is here not related to the Rayleigh speed. Capturing a potential oscillatory instability, which is found experimentally close to the Rayleigh speed in thin sheets [4], would require the consideration of asymmetric crack shapes.

Figure 3 shows the maximum height of the crack as function of the driving force for different loadings. At $\Delta \approx$ 1.1 the size of the mode III steady state crack diverges and $v \rightarrow 0$. The viscous dissipation becomes negligible here, but the surface dissipation remains finite. This point can be interpreted as the point of ductile-to-brittle transition: Below it the size grows indefinitely in time and the crack slows down, while above this point steady state solutions with a finite tip scale exist.

Starting from a pure mode III crack, we can now include additional mode I loadings. Figure 2 shows that this shifts the bifurcation point towards higher values and therefore extends the range of steady state solutions towards higher driving forces. Again, the crack blunts close to the "nominal" Griffith point $\Delta=1$. Simultaneously, the propagation velocity is significantly reduced in the regime of small $\Delta$, as can be clearly seen in the comparison between the cases with $15 \%$ and $85 \%$ mode I contribution. Effectively, this establishes an interval of driving forces, where the crack speed is very low, and only after this plateau it sharply increases; this effect becomes more pronounced as the crack loading is more mode I dominated. The same plateau can also be found in the tip scale; see Fig. 3.

For the case of mode I, finally, steady state solutions do not exist below $\Delta=2.6$; this result has to be interpreted as a limiting case with very slow creep with velocities and tip radii significantly lower than above the point $\Delta=2.6$. Literally, of course, growth starts at $\Delta=1$ due to energy conservation. The presence of this plateau is quite remark-

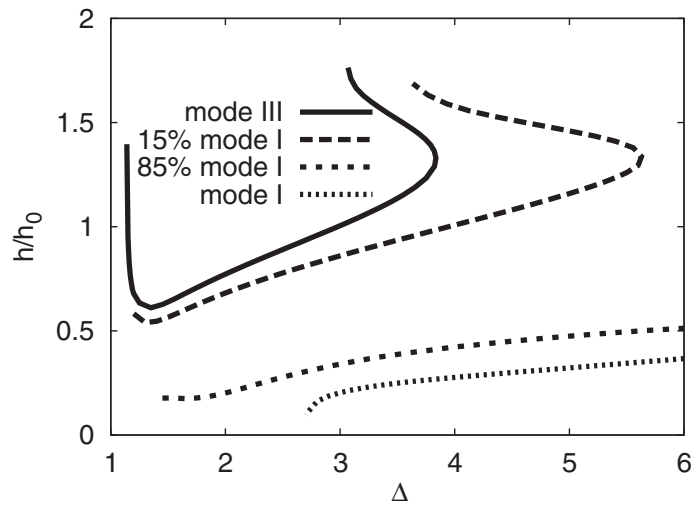

FIG. 3. Half crack height as a function of the driving force. The length scale used here is $h_{0}=\left(D \tau_{0}\right)^{1 / 4}$. 


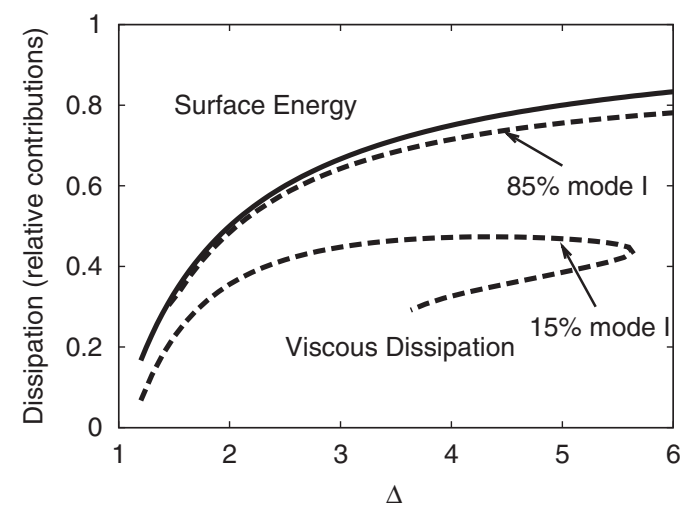

FIG. 4. Distribution of energy consumption. The fraction above the solid curve is the relative contribution of surface energy generation, the part below the dashed line the viscous dissipation (shown here for two different admixtures of mode I loading; for $15 \%$ mode I also for the unstable branch). The remaining part between the curves is the surface dissipation.

able, as this effectively renormalizes the "apparent" Griffith point - the driving force where the velocity starts to increase sharply - to a substantially higher value than $\Delta=1$, although the viscous dissipation remains finite on the "creep branch." Again, the crack speed increases monotonically with the driving force, and the bifurcation to unstable growth occurs only at very high driving forces.

Finally, Fig. 4 shows how the different mechanisms contribute to the total energy consumption: From the total dimensionless driving force the amount $\Delta-1$ is dissipated, and the remaining part is used for the creation of the surfaces of the advancing crack. Obviously, this contribution becomes less important in comparison to the true dissipation for higher driving forces. The viscous dissipation is

$$
\Delta_{v}=\frac{1}{2 \gamma v} \int_{V} R d V=\frac{1}{2 \gamma} \int_{S} \sigma_{\text {in }}^{(\mathrm{el})} u_{i, x} d s
$$

with the Rayleigh dissipation function $R=\sigma_{i k}^{(\text {vis })} \dot{\epsilon}_{i k}$, and the integration domain $V$ is the solid phase. The latter equality in Eq. (13) holds in steady state, where $S$ is the crack contour. The surface dissipation is

$$
\Delta_{s}=\frac{1}{4 \gamma} \int_{S} \sigma_{i k}^{(\mathrm{el})} \epsilon_{i k} n_{x} d s-1=\frac{v}{2 D} \int_{S} y^{2} d s,
$$

with the horizontal component of the interface normal $n_{x}$; again, the latter expression, which follows from Eq. (5), is valid only in the steady state regime. Altogether, we have $\Delta=\Delta_{s}+\Delta_{v}+1$.

Starting from the Griffith point the viscous dissipation continuously increases up to the point where the stable steady state solution branch terminates. It is quite remarkable, that for mode I dominated cracks the viscous dissipation $\Delta_{v}$ is much larger than $\Delta_{s}$, which shows that bulk dissipation can indeed play a crucial role. Notice that these (dimensionless) predictions do not depend on model parameters.

The obtained results lead to the striking conclusion, that the apparent Griffith point may depend quite substantially on the mode of loading. Although most models in the literature are discussed either in the mode I or mode III case only, we clearly see here that the behavior can be significantly different in these cases, as soon as bulk dissipation is taken into account. For the specific case of crack propagation in viscoelastic media we obtain that the onset of steady state growth is shifted towards higher values in mode I. It is therefore possible that local rotations or deformations of the crack front could lead to faster crack growth and a lower apparent Griffith point. We will leave this point, also in the context of the principle of local symmetry, open for future fully time-dependent threedimensional investigations.

In summary, we developed a model for crack propagation in viscoelastic media in the spirit of an interfacial pattern formation process. Motion occurs due to surface diffusion along the extended crack shape, which is-together with the propagation velocity and the tip scaleselected self-consistently. The steady state regime of the model is solved numerically using a series expansion method and a sharp interface description, which efficiently separates the microscopic crack tip scale from the system size. The results show that the bulk dissipation in the surrounding of the crack tip can play a substantial role especially for higher driving forces, and the crack velocity depends crucially on the mode of loading.

This work was supported by the German Research Foundation under grant SPP 1296 and the German-Israeli Foundation.

[1] R. J. Asaro and W. A. Tiller, Metall. Trans. 3, 1789 (1972).

[2] M. A. Grinfeld, Sov. Phys. Dokl. 31, 831 (1986).

[3] J. Fineberg and M. Marder, Phys. Rep. 313, 1 (1999).

[4] A. Livne, O. Ben-David, and J. Fineberg, Phys. Rev. Lett. 98, 124301 (2007).

[5] R. Spatschek, M. Hartmann, E. Brener, H. MüllerKrumbhaar, and K. Kassner, Phys. Rev. Lett. 96, 015502 (2006); D. Pilipenko, R. Spatschek, E. A. Brener, and H. Müller-Krumbhaar, Phys. Rev. Lett. 98, 015503 (2007); R. Spatschek, C. Müller-Gugenberger, E. Brener, and B. Nestler, Phys. Rev. E 75, 066111 (2007).

[6] T.-S. Lo, A. Pomyalov, I. Procaccia, and J. Zylberg, Phys. Rev. E 78, 027101 (2008).

[7] K. B. Broberg, Cracks and Fracture (Academic Press, London, 1999).

[8] B. N. J. Persson and E. A. Brener, Phys. Rev. E 71, 036123 (2005).

[9] L.D. Landau and E. M. Lifshitz, Theory of Elasticity (Pergamon Press, Oxford, 1987).

[10] M. Uwaha and P. Nozieres, J. Phys. (Paris) 47, 263 (1986). 